KYUNGPOOK Math. J. 48(2008), 317-321

\title{
Normal Families and Shared Values of Meromorphic Functions
}

\author{
Chao Meng
}

Department of Mathematics, Shandong University, Jinan 250100, Shandong, China e-mail : mengchao@mail.sdu.edu.cn

ABSTRACT. Some criteria for determining the normality of the family $F$ of meromorphic functions in the unit disc, which share values depending on $f \in F$ with their derivatives is obtained. The new results in this paper improve some earlier related results given by Pang and Zalcman [3], Fang and Zalcman [2], A. P. Singh and A. Singh [5].

\section{Introduction, definitions and main results}

Let $f$ and $g$ be meromorphic functions on a domain $D$ in $C$, and let $a$ and $b$ be complex numbers. If $g(z)=b$ whenever $f(z)=a$, we write

$$
f(z)=a \Rightarrow g(z)=b .
$$

In a different notation, we have $\bar{E}_{f}(a) \subset \bar{E}_{g}(b)$, where $\bar{E}_{h}(c)=h^{-1}(c) \cap D=\{z \in$ $D: h(z)=c\}$. If $f(z)=a \Rightarrow g(z)=b$ and $g(z)=b \Rightarrow f(z)=a$, we write

$$
f(z)=a \Leftrightarrow g(z)=b .
$$

If $f(z)=a \Leftrightarrow g(z)=a$, we say that $f$ and $g$ share $a$ on $D$.

Schwick is probably the first to find a connection between the normality criterion and shared values of meromorphic functions. He proved the following theorem.

Theorem $\mathbf{A}([4])$. Let $F$ be a family of meromorphic functions in the unit disc $\Delta$, and let $a_{1}, a_{2}, a_{3}$ be distinct complex numbers. If $f$ and $f^{\prime}$ share $a_{1}, a_{2}$ and $a_{3}$ for every $f \in F$, then $F$ is normal in $\Delta$.

Pang and Zalcman extended the above result as follows.

Theorem B([3]). Let $F$ be a family of meromorphic functions in the unit disc $\Delta$ and let $a$ and $b$ be distinct complex numbers and $c$ be a nonzero complex number. If for every $f \in F, f(z)=0 \Leftrightarrow f^{\prime}(z)=a$ and $f(z)=c \Leftrightarrow f^{\prime}(z)=b$, then $F$ is normal in $\Delta$.

Received December 18, 2007.

2000 Mathematics Subject Classification: 30D45, 30D35.

Key words and phrases: meromorphic function, shared value, normal family. 
In 2001, Fang and Zalcman proved the following result.

Theorem $\mathbf{C}([2])$. Let $F$ be a family of meromorphic functions in the unit disc $\Delta$ and let $b, c$ and $d$ be nonzero complex numbers such that $d \neq b$. Suppose that for each $f \in F, f(z)=0 \Rightarrow f^{\prime}(z)=b$ and $f^{\prime}(z)=d \Rightarrow f(z)=c$. Then $F$ is normal in $\Delta$ so long as $b \neq(m+1) d, m=1,2,3, \cdots$.

In Theorem $\mathrm{B}$ and Theorem $\mathrm{C}$ the constants are the same for each $f \in F$. In 2004, A. P. Singh and A. Singh proved that the condition for the constants to be the same can be relaxed to some extent. More precisely, they proved the following theorem.

Theorem $\mathbf{D}([\mathbf{5}])$. Let $F$ be a family of meromorphic functions in the unit disc $\Delta$. For each $f \in F$ let $a_{f}, b_{f}, c_{f}$ be distinct nonzero complex numbers such that $\left(a_{f} b_{f} / c_{f}^{2}\right)=M$ for some constant $M$. Let the spherical distance $\sigma$ between the points $a_{f}, b_{f}, c_{f}$ satisfy

$$
\min \left\{\sigma\left(a_{f}, b_{f}\right), \sigma\left(b_{f}, c_{f}\right), \sigma\left(c_{f}, a_{f}\right)\right\} \geq m
$$

for some $m>0$. Let $f(z)=0 \Leftrightarrow f^{\prime}(z)=a_{f}$ and $f(z)=c_{f} \Leftrightarrow f^{\prime}(z)=b_{f}$. Let $M=\left(a b / c^{2}\right)$, where $a, b, c$ are distinct. If the elements of $\bar{E}_{f}\left(c_{f}\right)$ and $\bar{E}_{f}(0)$ are the only solutions of

$$
f^{\prime}(z)=\frac{a_{f} b}{a}\left(1-\left(\frac{1}{c_{f}}-\frac{a}{c a_{f}}\right) f(z)\right)^{2}
$$

and

$$
f^{\prime}(z)=a_{f}\left(1-\left(\frac{1}{c_{f}}-\frac{a}{c a_{f}}\right) f(z)\right)^{2}
$$

respectively, then $F$ is normal in $\Delta$.

Now the following problem is considered: Is it possible to relax the nature of sharing values in Theorem D ? In this paper, we prove the following theorem which answers the above question.

Theorem 1. Let $F$ be a family of meromorphic functions in the unit disc $\Delta$. For each $f \in F$ let $a_{f}, b_{f}, c_{f}$ be distinct nonzero complex numbers such that $\left(a_{f} b_{f} / c_{f}^{2}\right)=M$ for some constant $M$. Let the spherical distance $\sigma$ between the points $a_{f}, b_{f}, c_{f}$ satisfy

$$
\min \left\{\sigma\left(a_{f}, b_{f}\right), \sigma\left(b_{f}, c_{f}\right), \sigma\left(c_{f}, a_{f}\right)\right\} \geq m
$$

for some $m>0$. Let $f(z)=0 \Rightarrow f^{\prime}(z)=a_{f}$ and $f^{\prime}(z)=b_{f} \Rightarrow f(z)=c_{f}$. Let $M=\left(a b / c^{2}\right)$, where $a, b, c$ are distinct and $a \neq(m+1) b, m=1,2,3, \cdots$. If the elements of $\bar{E}_{f}\left(c_{f}\right)$ are the only solutions of

$$
f^{\prime}(z)=\frac{a_{f} b}{a}\left(1-\left(\frac{1}{c_{f}}-\frac{a}{c a_{f}}\right) f(z)\right)^{2},
$$


then $F$ is normal in $\Delta$.

Remark. Theorem 1 removes the restriction on $\bar{E}_{f}(0)$ of Theorem $\mathrm{D}$ and the nature of sharing values is relaxed.

If the meromorphic functions in $F$ and their derivatives share $a_{f}$ bounded by some constant $M$, and $\alpha a_{f}$ respectively, then we shall prove the following theorem.

Theorem 2. Let $F$ be a family of meromorphic functions on the unit disc $\Delta$. For each $f \in F$ let there exist $a_{f}\left(0<\left|a_{f}\right| \leq M\right.$ for some constant $\left.M\right)$ and $\alpha a_{f}(\alpha \neq$ $1,2,3, \ldots$ is a constant) and $f(z)=a_{f} \Rightarrow f^{\prime}(z)=a_{f}, f^{\prime}(z)=\alpha a_{f} \Rightarrow f(z)=\alpha a_{f}$. Further let the elements of $\bar{E}_{f}\left(\alpha a_{f}\right)$ be the only solutions of

$$
f^{\prime}(z)=\frac{a_{f} b}{a}\left(1-A_{f}\left(f(z)-a_{f}\right)\right)^{2},
$$

where $A_{f}=\left(\left(1 /(\alpha-1) a_{f}\right)-\left(a / c a_{f}\right)\right), a$ is any nonzero constant, $b=\alpha a$ and $c=(\alpha-1) a$. Then $F$ is normal in $\Delta$.

\section{Some lemmas}

We need the following lemmas in the proof of Theorem 1 and Theorem 2 .

Lemma $\mathbf{1}([\mathbf{1}])$. The Mobius map $g(z)=\frac{a z+b}{c z+d}$, ad-bc=1 satisfies the Lipschitz condition

$$
\sigma(g(z), g(w)) \leq \frac{\pi}{2}\|g\|^{2} \sigma(z, w)
$$

where $\|g\|^{2}=|a|^{2}+|b|^{2}+|c|^{2}+|d|^{2}$.

The proof is immediate since from [5]

$$
\sigma_{0}(g(z), g(w)) \leq\|g\|^{2} \sigma_{0}(z, w)
$$

where $\sigma_{0}$ is the spherical metric, and the spherical metric and chordal metric $\sigma$ are related by

$$
\frac{2}{\pi} \sigma_{0}(z, w) \leq \sigma(z, w) \leq \sigma_{0}(z, w)
$$

Lemma 2([1]). Let $m$ be any positive number. Then the Mobius transformation $g$ which satisfies $\sigma(g(a), g(b)) \geq m, \sigma(g(b), g(c)) \geq m, \sigma(g(c), g(a)) \geq m$ for some constant $a, b$ and $c$, also satisfies the uniform Lipschitz condition

$$
\sigma(g(z), g(w)) \leq k_{m} \sigma(z, w)
$$

where $k_{m}$ is a constant depending on $m$. 


\section{Proof of Theorem 1}

For each $f \in F$, define a Mobius map $g_{f}$ by

$$
g_{f}(z)=\frac{z}{A z+B}
$$

where $A=\frac{1}{c_{f}}-\frac{a}{c a_{f}}$ and $B=\frac{a}{a_{f}}$. Then clearly we have

$$
g_{f}^{-1}(z)=\frac{B z}{1-A z}
$$

and

$$
\left(g_{f}^{-1}\right)^{\prime}(z)=\frac{B}{(1-A z)^{2}}
$$

so that $g_{f}^{-1}(0)=0, g_{f}^{-1}\left(c_{f}\right)=c,\left(g_{f}^{-1}\right)^{\prime}(0)=\frac{a}{a_{f}},\left(g_{f}^{-1}\right)^{\prime}\left(c_{f}\right)=\frac{b}{b_{f}}$.

Now if $z_{0}$ is such that $f\left(z_{0}\right)=0$ then since $f(z)=0 \Rightarrow f^{\prime}(z)=a_{f}$ we have $f^{\prime}\left(z_{0}\right)=a_{f}$ and so $\left(g_{f}^{-1} \circ f\right)\left(z_{0}\right)=g_{f}^{-1}(0)=0$ and $\left(g_{f}^{-1} \circ f\right)^{\prime}\left(z_{0}\right)=$ $\left(g_{f}^{-1}\right)^{\prime}\left(f\left(z_{0}\right)\right) f^{\prime}\left(z_{0}\right)=a$.

Now we show that $g_{f}^{-1} \circ f(z)=0 \Rightarrow\left(g_{f}^{-1} \circ f(z)\right)^{\prime}=a$. Let $z_{1}$ is such that $\left(g_{f}^{-1} \circ f\right)\left(z_{1}\right)=0$, then $\left(g_{f}^{-1} \circ f\right)\left(z_{1}\right)=0=g_{f}^{-1}(0)$. Also $g_{f}^{-1}$ being a Mobius map, is one-to-one so that $f\left(z_{1}\right)=0$ and so $f^{\prime}\left(z_{1}\right)=a_{f}$. Thus $\left(g_{f}^{-1} \circ f\left(z_{1}\right)\right)^{\prime}=a$.

Next we show that $\left(g_{f}^{-1} \circ f(z)\right)^{\prime}=b \Rightarrow g_{f}^{-1} \circ f(z)=c$. Let $z_{2}$ is such that $\left(g_{f}^{-1} \circ f\left(z_{2}\right)\right)^{\prime}=b$. Then $\left(g_{f}^{-1}\right)^{\prime}\left(f\left(z_{2}\right)\right) f^{\prime}\left(z_{2}\right)=b$ and so

$$
f^{\prime}\left(z_{2}\right)=\frac{a_{f} b}{a}\left(1-\left(\frac{1}{c_{f}}-\frac{a}{c a_{f}}\right) f\left(z_{2}\right)\right)^{2} .
$$

Since only the elements of $\bar{E}_{f}\left(c_{f}\right)$ satisfy (1), it follows that $f\left(z_{2}\right)=c_{f}$ and so $\left(g_{f}^{-1} \circ f\right)\left(z_{2}\right)=c$. Thus $\left(g_{f}^{-1} \circ f(z)\right)^{\prime}=b \Rightarrow g_{f}^{-1} \circ f(z)=c$.

Thus by Theorem $\mathrm{C}$, the family $G=\left\{\left(g_{f}^{-1} \circ f\right): f \in F\right\}$ is normal and hence equicontinuous in $\Delta$. Therefore given $\left(\epsilon / k_{m}\right)>0$, where $k_{m}$ is the constant of Lemma 2, there exist $\delta>0$ such that for the spherical distance $\sigma(x, y)<\delta$,

$$
\sigma\left(\left(g_{f}^{-1} \circ f\right)(x),\left(g_{f}^{-1} \circ f\right)(y)\right)<\frac{\epsilon}{k_{m}}
$$

for each $f \in F$. Hence by Lemma 2

$$
\begin{gathered}
\sigma(f(x), f(y))=\sigma\left(\left(g_{f} \circ g_{f}^{-1} \circ f\right)(x),\left(g_{f} \circ g_{f}^{-1} \circ f\right)(y)\right) \\
\leq k_{m} \sigma\left(\left(g_{f}^{-1} \circ f\right)(x),\left(g_{f}^{-1} \circ f\right)(y)\right)<\epsilon .
\end{gathered}
$$


Thus the family $F$ is equicontinuous in $\Delta$. This completes the proof of Theorem 1 .

\section{Proof of Theorem 2}

Let $b_{f}=\alpha a_{f}$ and $c_{f}=(\alpha-1) a_{f}$ so that $a_{f} b_{f} / c_{f}^{2}$ is the constant $\alpha /(\alpha-1)^{2}$. For each $f \in F$, define $g_{f}(z)=f(z)-a_{f}$. Let $G=\left\{g_{f}: f \in F\right\}$. Then clearly

$$
g_{f}(z)=0 \Rightarrow f(z)=a_{f} \Rightarrow f^{\prime}(z)=a_{f} \Rightarrow g_{f}^{\prime}(z)=a_{f}
$$

and

$$
g_{f}^{\prime}(z)=b_{f} \Rightarrow f^{\prime}(z)=b_{f} \Rightarrow f(z)=b_{f} \Rightarrow g_{f}(z)=c_{f} .
$$

From the assumption of Theorem 2, we get that the elements of $\bar{E}_{g_{f}}\left(c_{f}\right)$ are the only solutions of

$$
g_{f}^{\prime}(z)=\frac{a_{f} b}{a}\left(1-A_{f} g_{f}(z)\right)^{2} .
$$

Hence by Theorem $1, G$ is normal in $\Delta$, and hence given $\epsilon>0$, there exists $\delta>0$ for all $x, y$ such that $\sigma(x, y)<\delta$, then we have $\sigma\left(g_{f}(x), g_{f}(y)\right)<\epsilon$ for every $f \in F$. Now define $R_{f}(z)=z+a_{f}$, then each $R_{f}(z)$ is a Mobius map and $R_{f}\left(g_{f}(z)\right)=f(z)$. Hence by Lemma 1

$$
\begin{aligned}
\sigma(f(x), f(y)) & =\sigma\left(R_{f}\left(g_{f}(x)\right), R_{f}\left(g_{f}(y)\right)\right) \\
& \leq\left\|R_{f}\right\|^{2} \frac{\pi}{2} \sigma\left(g_{f}(x), g_{f}(y)\right) \\
& \leq\left(2+M^{2}\right) \frac{\pi}{2} \epsilon .
\end{aligned}
$$

Hence $F$ is normal in $\Delta$. This completes the proof of Theorem 2 .

\section{References}

[1] A. F. Beardon, Iteration of Rational Functions, Springer-Verlag, New York, 1991.

[2] M. L. Fang and L. Zalcman, Normal families and shared values of meromorphic functions II, Comput. Methods Funct. Theory, 1(2001), 289-299.

[3] X. C. Pang and L. Zalcman, Normality and shared values, Arkiv Math., 38(2000), 171-182.

[4] W. Schwick, Sharing values and normality, Arch Math.(Basel), 59(1992), 50-54.

[5] A. P. Singh and A. Singh, Sharing values and normality of meromorphic fucntions, Complex Variables Theory Appl., 49(2004), 417-425. 